\title{
Loss of Complexity of the Cardiac Bioelectrical Signal as an Expression of Patient Outcomes
}

\author{
Pedro Eduardo Alvarado Rubio, \\ Ricardo Mansilla Corona, Lizette Segura Vimbela, \\ Alejandro González Mora, Roberto Brugada Molina, \\ Cesar Augusto González López and \\ Laura Yavarik Alvarado Avila
}

Additional information is available at the end of the chapter

http://dx.doi.org/10.5772/intechopen.70144

\begin{abstract}
The loss of complexity of the cardiac bioelectrical signal, measured with tools of nonlinear dynamics (NLD), is studied in patients with very different pathologies. Two types of scenarios are studied: (a) patients who enter the critical care unit and recover from their condition; (b) severe patients whose condition worsen and finally die. It is shown that as the severity of the patients increases, the complexity of their cardiac bioelectric signal decreases. On the other hand, if patients, despite being severe, manage to recover, the cardiac bioelectric signal recovers its complexity.
\end{abstract}

Keywords: bioelectrical signal, nonlinear dynamics, complexity, variability, critical illness

\section{Introduction}

The application of tools of the theory of the dynamical systems to the study of physiological phenomena has a long inheritance. This starts from the original works of van der Pol and his collaborator [1, 2], passes through important contributions [3, 4], and reaches the comprehensive work by Glass and Mackey [5]. The application of these types of systems to describe the temporal evolution of the physiological phenomena has been established as a tool frequently used by researchers in this area of knowledge and is already a common place in the literature.

The initial works in the analysis of the dynamics of the cardiac rhythm showed a nonlinear dynamic (NLD) behavior. Period-doubling bifurcations, in which the period of a regular oscillation doubles, were predicted theoretically and observed experimentally in the heart cells of 
embryonic chickens [6]. The tools with a new mathematical approach made it possible to apply the nonlinear dynamics to basic physiological concepts, proving for the first time evidence of nonlinear behavior in the electrocardiogram (ECG) [7]. Period multiplying evidence in arterial blood pressure traces of a dog that had been injected with noradrenaline was reported in 1984 [8]. Since the original reports on ischemic heart disease and arrhythmia $[9,10]$, the analysis of spontaneous variations of beat-to-beat intervals (BBIs) has become an important clinical tool [11-13].

For a long time, the construction of models was based on first principles, being this the main tool for the understanding of complex physiological phenomena in theoretical models of nerve and the membrane $[14,15]$, among which the functioning of the heart occupies a main place. On the other hand, some studies tried to obtain information about cardiac diseases from the time series that the measuring instruments offered, although very often they were noisy and limited data [16]. Procedures have been developed for the study of ventricular fibrillation using the data of implantable defibrillators $[13,17]$. It is well known that these data are often noisy and only represent the sequence of $R-R$ intervals and is typically morphologically different from surface electrocardiogram recordings. Many signal-processing algorithms have been designed to eliminate noise from a system; however, noise (i.e., stochastic processes) is a critical component of many biological and physiological systems [18]. Given the difficulties mentioned earlier, some authors tried to use measures of complexity and entropies, as well as other techniques of the theory of nonlinear phenomena $[19,20]$.

The initial clinical observation of heart rate variability (HRV), observing changes in the pattern of the R-R interval, which preceded changes in the heart rate in fetal distress, was reported in 1963 [21]. Later, the first approaches of the heart rate variability analysis based on nonlinear fractal dynamics were performed in 1987 [4]. It was suggested that self-similar (fractal) scaling may underlie the 1/f-like spectra [22] seen in multiple systems (e.g., interbeat interval variability, daily neutrophil fluctuations). They proposed that this fractal scale invariance may provide a mechanism for the "constrained randomness" underlying physiological variability and adaptability. In 1988, it was reported that patients prone to high risk of sudden cardiac death showed evidence of nonlinear heart rate (HR) dynamics, including abrupt spectral changes and sustained low-frequency (LF) oscillations. After this report, it has been suggested that a loss of complex physiological variability could occur under certain pathological conditions such as reduced HR dynamics before sudden death and aging [23, 24].

Methods of NLD and fractal analysis have opened up new ways for the analysis of HRV. Although time and frequency domain methods enable the quantification of HRV on different time scales, nonlinear methods provide additional information regarding the dynamics and structure of beat-to-beat time series in various physiological and pathophysiological conditions [25]. The apparent loss of multiscale complexity in life-threatening conditions suggests a clinical importance of this multiscale complexity measure. Studies on heart rate multiscale entropy at $3 \mathrm{~h}$ predict hospital mortality in patients with major trauma [26]. Joint symbolic dynamics, compression entropy, fractal dimension, and approximate entropy revealed significantly reduced complexity of heart rate time series and loss of efferent vagal activity in acute schizophrenia $[27,28]$.

The healthy human heart rate is mainly determined by three major inputs: the sinoatrial node; and the parasympathetic and sympathetic branches of the autonomous nervous system and several autocrine, paracrine, and endocrine substances effects on it [29]. The sinoatrial node or 
pacemaker is responsible for the initiation of each heart beat; in the absence of other external stimuli, it is able to maintain an essentially constant interbeat interval. Experiments in which parasympathetic and sympathetic inputs are blocked reveal that the interbeat intervals are very regular and average $0.6 \mathrm{~s}$. The parasympathetic fibers conduct impulses that increase the interbeat intervals. Suppression of sympathetic stimuli, while under parasympathetic regulation, can result in the increase of the interbeat interval to as much as $1.5 \mathrm{~s}$. The activity of the parasympathetic system changes with external stimuli and with internal cycles. The sympathetic fibers conduct impulses that decrease the interbeat intervals. Abolition of parasympathetic influences when the sympathetic system remains active can decrease the interbeat intervals to less than $0.3 \mathrm{~s}$. There are several centers of sympathetic activity which are highly sensitive to environmental influences [30,31]. All the patients that are analyzed in this work have as a common factor a suffering of systemic repercussion that influences the dynamics of the sympathetic-parasympathetic balance and, therefore, of the heart rate. As will be seen subsequently, whatever the underlying condition, as it worsens, decreases the complexity of the cardiac bioelectrical signal, while its improvement is accompanied by an increase in the complexity of the cardiac signal. Hence, the measures of complexity of the heart electrical signals allow assessing the severity of the patient's condition, and as we will see later (Figure 3) they can be used as early warnings of severity episodes. This is why we focus on the observation of the complexity of the cardiac bioelectric signal.

The increasing availability of physiological data has allowed the study of long-time series with other techniques also coming from the theory of dynamical systems. The observations received from some organ of our body, as we have said before, very often are tainted of noise or are collected in an incomplete way. The signals of electrical activity of the heart are a good example of this. They are measured on the surface of the patient, in a finite number of places. The electrical signal from the heart must pass through several layers of tissues with different electrical conductivities before being measured by traditional devices. What we measure is actually a distorted observable of the authentic electric signal coming from the surface of the heart. Then the following question arises; how much information of the original phenomenon could be recovered from this distorted signal? Other relevant questions are how many magnitudes are necessary for a complete description of the phenomenon? In other words, what is the dimension of the attractor of the dynamical system that describes the evolution of the heart? Is there a difference between a healthy person and a sick person in the number of variables necessary to characterize their behavior? Or put in another way, is there a difference in the dimension of the attractor of the dynamical systems that describe the behavior of a healthy person and a sick person?

The Takens Embedding Theorem [32] answers these questions under certain assumptions about the recorded time series. In the following lines, we describe the theoretical framework, the fundamental results, and the techniques of valuation of the different magnitudes.

\section{Mathematical theoretical framework}

The most frequent problems in the study of the physiological signals of electrical type are that very often we have incomplete and deformed information of them. This is a very common problem in many branches of scientific knowledge. As far as the electrical activity of the 
cardiac muscle is concerned, a methodology has been developed, whose theoretical basis is found in the theory of dynamical systems. This result is known as the Takens' Embedding Theorem. This result allows us, under certain hypotheses, to answer the following questions: Is it possible to reconstruct the bioelectric dynamics of a cardiac phenomenon from incomplete information? How many variables are necessary to fully characterize this phenomenon?

Let us assume that $n$ measurements would be necessary to fully characterize the phenomenon under study. We do not know them directly. Rather, we have a macroscopic observable $g$ that is constructed from them:

$$
g(t)=\theta\left(x_{1}(t), \ldots, x_{n}(t)\right)
$$

Here, we assume that $x_{1}(t), \ldots, x_{n}(t)$ are the measurements necessary to characterize the system, measured at time $t$. The function $\theta$ is one that transforms the variables that characterize the system in the macroscopic observable to which we have access.

Almost all medical devices discreetly take samples with a certain frequency. Therefore, what in the practice we have is a time series $\left\{g_{1}, \ldots, g_{T}\right\}$, where $g_{i}=g\left(t_{i}\right)$, where very often it is assumed (as do we) that the times of measurement $t_{i}$ are equally spaced in time.

The Takens Embedding Theorem basically says that under the assumption that function $g$ is "well behaved," that is, which can be measured continuously without very sudden jumps then, there are $\tau \in R^{+}$and $N \in N$ such that the set of vectors:

$$
A_{d}=\left\{\left(g_{i}, g_{i+\tau}, \ldots, g_{i+(N-1) \tau}\right), i \in N\right\}
$$

is for any practical purpose similar in its properties to the simultaneous behavior of the variables $x_{1}(t), \ldots, x_{n}(t)$. Unfortunately, the theorem does not say how $\tau$ and $N$ should be calculated. So a wide heuristic has been developed to estimate these parameters [33]. In this task, two concepts play an important role: the mutual information function and the correlation integral.

The mutual information function can be defined as follows:

$$
M(\tau)=\sum_{t=1}^{T-\tau} P\left(g_{t}, g_{t+\tau}\right) \ln \left[\frac{P\left(g_{t}, g_{t+\tau}\right)}{P\left(g_{t}\right) P\left(g_{t+\tau}\right)}\right]
$$

This concept is closely related to the concept of Boltzmann entropy and the Shannon information [34]. It is a measure of nonlinear correlation among the values of the series $\left\{g_{i}\right\}$.

The values of the mutual information function can be calculated from the $\left\{g_{1}, \ldots, g_{T}\right\}$ series using appropriate software. The correct value for $\tau$ is the first local minimum of the mutual information function $[15,35]$. It is well known that the mutual information function is more sensitive to correlations of data than other correlation measures [36].

The second important step is the calculation of the so-called embedding dimension N. For this, it is necessary to use a concept called integral correlation: consider now the collection of vector of the set $A_{d}$ : 


$$
X_{i}=\left(g_{i}, g_{i+\tau^{\prime}}, \ldots, g_{i+(N-1) \tau}\right)
$$

the correlation integral $C_{m}(\varepsilon)$ is defined as

$$
C_{m}(\varepsilon)=\left(\begin{array}{c}
m \\
2
\end{array}\right)^{-1} \sum_{1 \leq i, j \leq m} H\left(\left\|X_{i}-X_{j}\right\|<\varepsilon\right)
$$

where $H$ is the Heaviside function. From this concept, we can define the correlation dimension as

$$
d_{c}=\lim _{\varepsilon \rightarrow 0} \lim _{m \rightarrow+\infty} \frac{\ln C_{m}(\varepsilon)}{\ln \varepsilon}
$$

Now, the criteria for selecting the correct embedding dimension $N$ are as follows: choose increasing embedding dimension and in each case, calculate the correlation integral. When no changes are observed in the behavior of the correlation integral with respect to increasing the embedding dimension, then a suitable dimension immersion $[15,33]$ will be found.

One of the advantages of this method is its robustness with respect to the noise of the signal under study. The numerical data obtained through a recording apparatus, in our case a Holter, are the basis of all the further calculations in this chapter. Despite the fine structures of the cardiac dynamics, a critical component of many biological and physiological systems [19], could be lost in conventional Holters [3], the attractor of the system is reconstructed with adequate embedding and correlation dimensions.

The entire process can be seen in Figure 1.

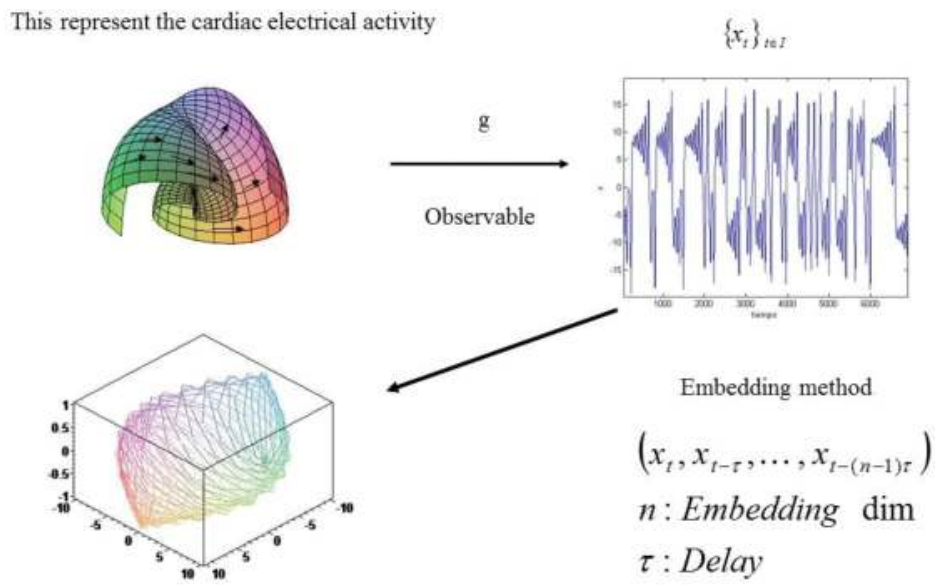

This represent the reconstruction of the atractor from Taken's Theorem

Figure 1. A representation of the entire embedding process. The phenomenon under study has an attractor which only has incomplete information through an observable. From this observable, the immersion process is executed, creating a reconstruction of the attractor. 


\section{Method}

\subsection{Description of the patients under study}

We have studied the biological electrical signal of electrocardiogram assuming that its evolution is governed by a dynamic system. A three-channel, 1-h Holter (Scott Care Corporation. Chroma: Model RZ153C) Monitor was used to monitor 30 patients to obtain data files which consist of 900,000 rows and three columns of comma separated values from $1 \mathrm{~h}$ of registration. Holter monitoring was performed on each patient every $24 \mathrm{~h}$ for a period of $1 \mathrm{~h}$ from admission until discharge. All admission Holter records were performed with the sedated patients, with amines and ventilatory mechanical support in the supine position. The Holter records of the surviving patients also are performed in supine position, without mechanical ventilation or cardiovascular support with amines. The diagnosis of patients was performed from the clinical point of view and confirmed by imaging studies such as computed tomography (CT) of the site topologically involved, such as computed axial tomography of the skull, chest, or abdomen. Some of the patients were surgically operated on one or more occasions. We studied 30 critically ill patients (18 females, 12 male age $54.8 \pm 15.3$ years old) of various pathologies, qualified with APACHE II scale $29.26 \pm 3.16$ on admission to intensive care.

The APACHE II Severity of Disease Classification System

\begin{tabular}{|c|c|c|c|c|c|c|c|c|c|}
\hline $\begin{array}{l}\text { Physiologic } \\
\text { Variable }\end{array}$ & +4 & +3 & +2 & +1 & 0 & +1 & +2 & +3 & +4 \\
\hline $\begin{array}{c}\text { Temperature - } \\
\text { rectal }(\mathrm{C})\end{array}$ & 241 & $39-40.9$ & & 38.5 .38 .9 & $36-38.4$ & $34-35.9$ & $32-33.9$ & $30-319$ & $\$ 29.9$ \\
\hline $\begin{array}{l}\text { Mean Arterial } \\
\text { Pressure (mm Ho) }\end{array}$ & $\geq 160$ & $130-159$ & $110-129$ & & $70-109$ & & $50-69$ & & $\leq 49$ \\
\hline Heart Rate & 2180 & $140-179$ & $110-139$ & & $70-109$ & & $55-69$ & $40-54$ & $\leq 99$ \\
\hline $\begin{array}{c}\text { Respiratory Rate } \\
\text { (posventilased or } \\
\text { ventiluted) }\end{array}$ & 250 & $35-49$ & & $25 \cdot 34$ & $12-24$ & $10-11$ & $\leftrightarrow$ & & ss \\
\hline \multirow{2}{*}{ 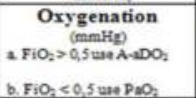 } & 2500 & $350-499$ & $200-349$ & & \multicolumn{5}{|l|}{$<200$} \\
\hline & \multicolumn{4}{|l|}{$b$} & $>70$ & $01-70$ & & $55-60$ & ess \\
\hline Arterial $\mathrm{pH}$ & 27.7 & $7.6-7.69$ & & $7.5-7.59$ & $7.33-7.49$ & & $7.25-7.32$ & $7.15-7.24$ & $<7.15$ \\
\hline Serum Sodium & 2180 & $160-179$ & $155-159$ & $150-154$ & $130-149$ & & $120-129$ & $111-119$ & $\leq 110$ \\
\hline $\begin{array}{c}\text { Serum Potassium } \\
\text { (mmol } 1)\end{array}$ & 27 & 0.6 .9 & & 5.5 .5 .9 & $3.5-5.4$ & $3-3.4$ & 25.29 & & 223 \\
\hline $\begin{array}{l}\text { Serum Creatinine } \\
\text { (ms d1, Double point } \\
\text { scoes for acute renal } \\
\text { fallure) }\end{array}$ & 23.5 & $2-3.4$ & $1.5 \cdot 1.9$ & & $0.0-1.4$ & & $<0.6$ & & \\
\hline Hematocrit (9o) & 260 & & 50.59 .9 & $46-49.9$ & 30.459 & & $20-29.9$ & & $<20$ \\
\hline $\begin{array}{l}\text { White Blood } \\
\text { Count is } 1000 \mathrm{~mm} \text { ? }\end{array}$ & $\geq 40$ & & $20-39.9$ & $15-19.9$ & 3.14 .9 & & 1.29 & & $<1$ \\
\hline $\begin{array}{l}\text { Glasgow-Coma- } \\
\text { Scale (CCS) }\end{array}$ & \multicolumn{9}{|c|}{ Score $=15$ minua acrual CCS } \\
\hline $\begin{array}{c}\text { Serum } \mathrm{HCO}_{3} \\
\text { (venowa, mmol1, we if no } \\
\mathrm{ABCy} \text { ) }\end{array}$ & 252 & 41.51 .9 & & $32-40.9$ & 22.31 .9 & & $18-219$ & $15-17.9$ & $<15$ \\
\hline $\begin{array}{c}A=\text { Total Acute } \\
\text { Physiology Score } \\
\text { APS }\end{array}$ & \multicolumn{9}{|c|}{ Sum of the 12 individual variable points } \\
\hline $\mathrm{B}=$ Age Points & \multicolumn{9}{|c|}{$\mathrm{C}=$ Chronic Health Points } \\
\hline 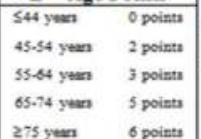 & $\begin{array}{c}\text { If the } \\
\text { immunc } \\
\text { b. }\end{array}$ & $\begin{array}{l}\text { patient } \\
\text { fomprom } \\
\text { For nooop } \\
\text { For telctiv }\end{array}$ & $\begin{array}{l}\text { has a } \\
\text { ised assig } \\
\text { nave or en } \\
\text { pontopernt }\end{array}$ & $\begin{array}{l}\text { istory } \\
\text { a points a } \\
\text { rgecy pos } \\
\text { ve patients }\end{array}$ & $\begin{array}{l}\text { severe } \\
\text { follows: } \\
2 \text { points }\end{array}$ & organ & stem in & ufficienc & or is \\
\hline
\end{tabular}

(From: Knaus WA, Draper EA, Wagner DP, Zimmerman JE. APACHE II: a severity of disease classification system. Crit Care Med 1985;13(10):818-29) 
The vital signs on the admission of these patients were as follows: Heart rate, $91.6 \pm 17.51$; respiratory rate, $21.6 \pm 6.43$; mean arterial pressure, $71.4 \pm 20.8$; and temperature of $37.6 \pm 1.3$.

Seventy percent of the patients were admitted with cardiovascular support based on noradrenaline infusion and sedated with ventilatory mechanical support. The nonlinear time series [33] were obtained upon admission to intensive care in the morning every day, until their discharge for improvement or death. The numerical data obtained from the comma-separated values by means of the Holter are the data subject to analysis of each patient. The description of patients is shown in Table 1.

\begin{tabular}{|c|c|c|c|c|c|c|}
\hline$N^{\circ}$ & Age & Sex & Diagnosis & Surgery $N^{\circ}$ & APACHE II/\% & Survived/died \\
\hline 1 & 64 & M & Severe post traumatic cerebral edema & Yes/1 & $27 / 55 \%$ & Died \\
\hline 2 & 55 & $\mathrm{~F}$ & $\begin{array}{l}\text { Cerebral Hemorrhage Fisher IV Malformation } \\
\text { Arterio/Venous }\end{array}$ & Yes/1 & $24 / 40 \%$ & Died \\
\hline 3 & 60 & M & Necrotizing Pancreatitis & Yes/2 & $30 / 75 \%$ & Died \\
\hline 4 & 34 & $\mathrm{~F}$ & $\begin{array}{l}\text { Traumatic Brain Injury - Intraparenchymal } \\
\text { Hemorrhage }\end{array}$ & Yes/1 & $20 / 40 \%$ & Survived \\
\hline 5 & 78 & $\mathrm{~F}$ & $\begin{array}{l}\text { Fisher IV Brain Hemorrhage by Ruptured Cerebral } \\
\text { Aneurism }\end{array}$ & Yes/1 & $29 / 55 \%$ & Died \\
\hline 6 & 23 & M & Wounded by gun fire in right eye & No & $28 / 55 \%$ & Died \\
\hline 7 & 30 & $\mathrm{~F}$ & $\begin{array}{l}\text { Postpartum complicated-Eclampsia and } \\
\text { Pulmonary Embolism }\end{array}$ & Yes/1 & $27 / 55 \%$ & Survived \\
\hline 8 & 26 & $\mathrm{M}$ & Septic shock by Appendicitis complicated & Yes/3 & $32 / 75 \%$ & Died \\
\hline 9 & 52 & M & Cerebellar Infarction & Yes/1 & $28 / 55 \%$ & Died \\
\hline 10 & 52 & $\mathrm{~F}$ & Septic Shock of Abdominal Origin & Yes/4 & $30 / 75 \%$ & Died \\
\hline 11 & 34 & M & Colon Necrosis and Septic Shock & Yes/3 & $29 / 55 \%$ & Survived \\
\hline 12 & 69 & $\mathrm{~F}$ & Epidural Hematoma & Yes/1 & $25 / 55 \%$ & Died \\
\hline 13 & 40 & $\mathrm{M}$ & Atypical Pneumonia. Acquired Immune Deficiency & No & $35 / 85 \%$ & Died \\
\hline 14 & 56 & M & Septic Shock-Pneumonia & No & $32 / 75 \%$ & Died \\
\hline 15 & 59 & M & Pulmonary Embolism & No & $30 / 75 \%$ & Died \\
\hline 16 & 78 & $\mathrm{~F}$ & Subarachnoid Hemorrhage-Aneurysm Rupture & Yes/1 & $29 / 55 \%$ & Died \\
\hline 17 & 63 & M & Acute Myocardial Infarction & No & $27 / 55 \%$ & Died \\
\hline 18 & 59 & M & Pulmonary Embolism. & No & $30 / 75 \%$ & Survived \\
\hline 19 & 47 & $\mathrm{~F}$ & Post Cardiorespiratory. Arrest Trans Surgical & Yes/1 & $32 / 75 \%$ & Died \\
\hline 20 & 74 & $\mathrm{~F}$ & Pulmonary Embolism. & No & $27 / 55 \%$ & Died \\
\hline 21 & 62 & $\mathrm{~F}$ & Pulmonary Embolism. & No & $28 / 55 \%$ & Died \\
\hline 22 & 62 & M & Left cerebellar hemisphere infarction. & Yes/1 & $32 / 75 \%$ & Survived \\
\hline 23 & 47 & $\mathrm{~F}$ & Cerebral Hemorrhage - Broken Aneurysm & Yes/1 & $29 / 55 \%$ & Died \\
\hline 24 & 43 & $\mathrm{~F}$ & Cerebral Hemorrhage-Broken Aneurysm & Yes/1 & $29 / 55 \%$ & Died \\
\hline 25 & 73 & $\mathrm{~F}$ & $\begin{array}{l}\text { Traumatic Brain Injury - Intraparenchymal } \\
\text { Hemorrhage }\end{array}$ & Yes/1 & $30 / 75 \%$ & Died \\
\hline
\end{tabular}




\begin{tabular}{lllllll}
\hline$N^{\circ}$ & Age & Sex & Diagnosis & Surgery $N^{\circ}$ & APACHE II/\% & Survived/died \\
\hline 26 & 60 & F & Hemorrhage Fisher III. Flegmásia Cerulea Dolens & No & $35 / 85 \%$ & Died \\
27 & 42 & F & Hemorrhage Subarachnoid Fisher IV & Yes $/ 1$ & $30 / 75 \%$ & Died \\
28 & 62 & F & Fisher IV Brain Hemorrhage & No & $29 / 55 \%$ & Died \\
29 & 71 & F & Cerebral Stroke & No & $30 / 75 \%$ & Died \\
30 & 69 & F & Traumatic Brain Injury - Intraparenchymal & Yes/1 & $35 / 85 \%$ & Died \\
& & & & & & \\
\end{tabular}

Table 1. Estimated mortality in critical patients according with APACHE II score.

The images show a CT scan where two images of patient No. 22 are shown of the posterior fossa of a 62-year-old man with hypertension treated with captopril $25 \mathrm{mg}$ every $12 \mathrm{~h}$. IMAGE "A" shows a hypodense zone of the left cerebellar hemisphere, suggesting an ischemic type lesion. IMAGE " $\mathrm{B}$ " shows decompressive craniotomy. The patient on day 10 of intensive care was without mechanical ventilation and interacting with the staff of the unit. As mentioned previously in the text, measures of the complexity of their cardiac signal were lower at the time of entry to the intensive care unit, which was recovered. Later, he was discharge from the critical medicine unit.
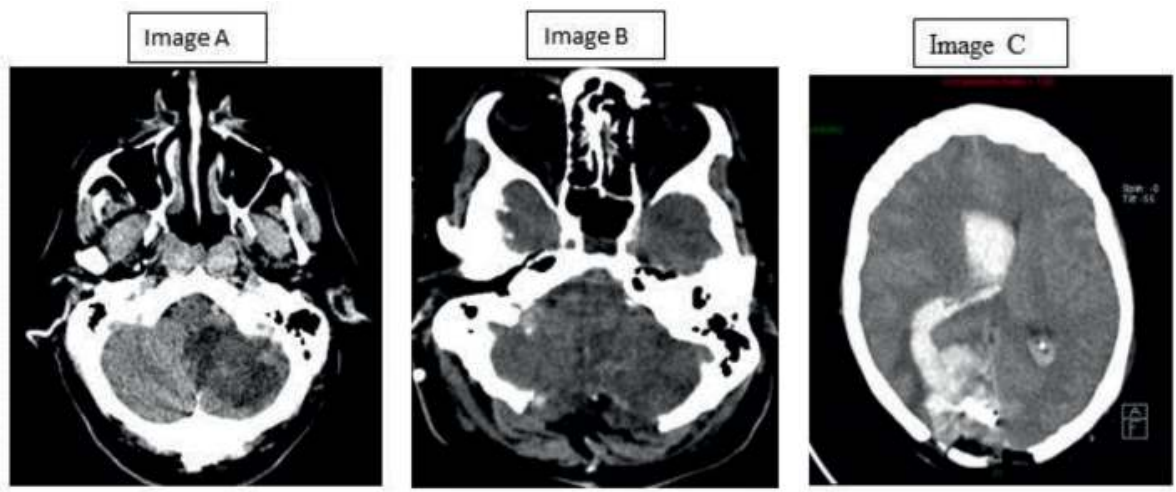

IMAGE "C" displayed is a cut of the cranial computed tomography of a 56-year-old woman with right occipital arteriovenous malformation. The patient during her stay in intensive care was complicated by acute myocardial infarction, cardiogenic shock, dying 12 days after her admission.

\section{Results}

Let us consider a moving window as shown in Figure 2.

For each moving windows, the correct value of $\tau$ and the embedding dimension $N$ are calculated. Note that the last task means calculating the correlation integral for different dimensions of immersion, as shown in Figure 2. 


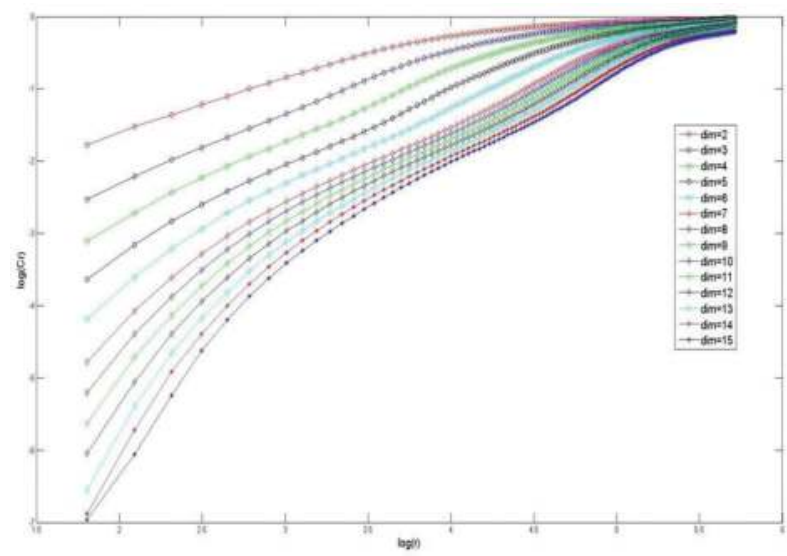

Figure 2. Correlation integrals for different embedding dimensions.

Once the appropriate dimension of embedding for each mobile window was calculated, we calculated the corresponding correlation dimension. In total, between sick and healthy people we amount 96,508 mobile windows of 5000 points each. We decided to choose the length of the mobile window equal to 5000 because we have observed that for that distance the average value of the mutual information function is practically zero, which indicates that over the time series, values separated by 5000 units of time or more have any correlation.

With these data, we calculate the probability density functions of the corresponding windows for healthy and sick behaviors. The results appear in Figure 3.

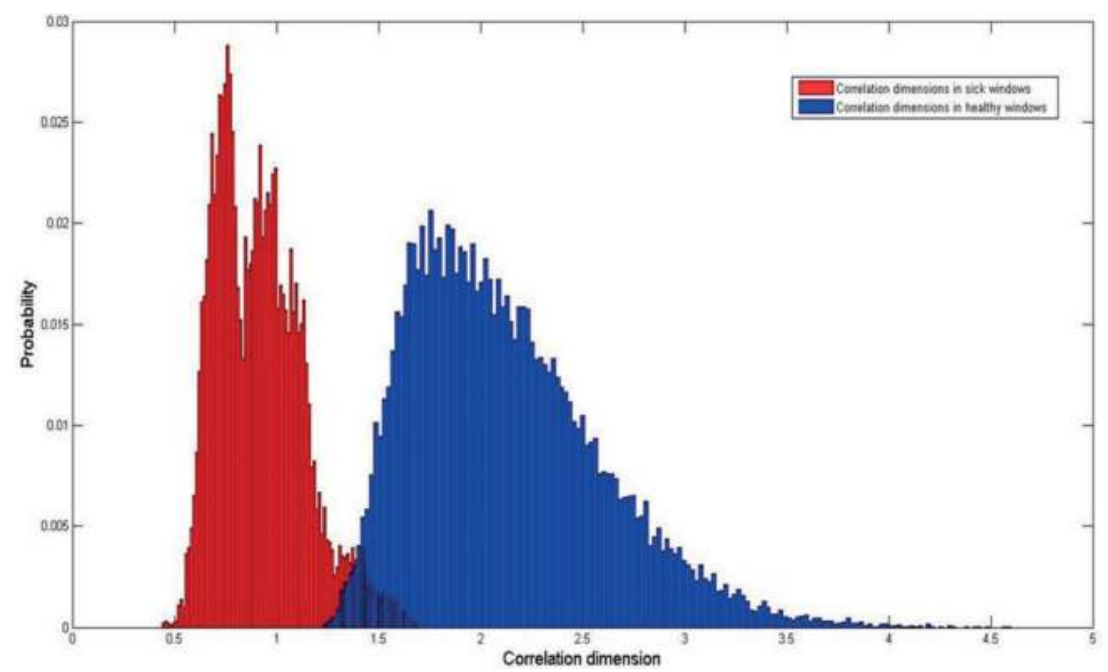

Figure 3. Probability density functions of correlation dimensions for windows sick (clearer) behavior an healthy (darker) behavior. 
Note that there is a clear separation between the dimensions of correlation for sick and healthy behaviors. Finally, we note that small-dimensional correlation magnitudes are related to less complex behaviors than those with high correlation dimensions. It is important to note that in our database are records of people who were healthy and became in serious condition, as well as people who were sick and recovered later.

\section{Discussion}

The neural regulation of cardiac bioelectric signal has been explored in the frequency domain, showing the complexity of the sympathovagal balance which is tonically and phasically modulated by the interaction of at least three major factors: (a) central neural integration, (b) peripheral inhibitory reflex mechanisms (with negative-feedback characteristics), and (c) peripheral excitatory reflex mechanisms (with positive-feedback characteristics) [29]. Parasympathetic efferent nerve fibers come through the parasympathetic ganglia positioned in the periaortic and epicardial fat pad. Efferent sympathetic innervation arrives from superior, middle, and inferior cervical and the upper four or five thoracic ganglia. Medullary nuclei and reticular formation give both excitatory and inhibitory preganglionic efferent fibers as well as accept afferent fibers. Afferent pathways from baroreceptors and the so-called cardiopulmonary receptors to the brain stem are closing the loop assuring a feedback mechanism. These baroreceptors are located in the wall of aortic arch and great arteries arising from it, and in the carotid sinus. The hypothalamus is considered the most important supramedullary compound that integrates autonomic, somatic, mental, and emotional information via its extensive associations [29].

The applications of entropy on human physiological signals were developed earlier for analyzing the heart rate and beat-to-beat blood pressure. Heart rate is influenced by numerous factors including the liquid metabolism, hormonal and temperature variations, physical activity, circadian rhythms, and autonomic nervous system. As a result, heart rate variations are extremely complex in healthy individuals [37].

The entropy of heart rate was linked to neurological system since the modulation of heart beat was associated with the two components of autonomic nervous system: sympathetic and parasympathetic nerves [38]. Rhythmical oscillations of both heart rate and blood pressure have been indicated to reflect the sympathetic and parasympathetic modulation $[39,40]$.

The evaluation of the autonomic function provides important information about the alteration of the sympathetic-parasympathetic, altered balance in critically ill patients with or without multiple organ failure. The proven tools are heart rate variability, baroreflex sensitivity, and, with limitations, cardiac chemoreflex sensitivity [38]. The R-R interval functions as a substitute for the sympathovagal balance and represents the net result of all autonomic influences in the sinus node. Both the sympathetic and parasympathetic inputs to the sinus node can be characterized by a tonic level of activity and by the modulation of this activity (e.g., by respiration in the case of the parasympathetic input). Heart rate variability most reliably provides a 
measure of the modulation of the sympathetic and parasympathetic inputs to the sinus node, although a more precise way to characterize the sympathovagal balance is unknown [41, 42]. With new, nonlinear methods being evaluated, the risk (with prognostic implications) could be predicted more accurately by providing additional information on autonomic heart rate control in critically ill patients [29].

\section{Conclusions}

The results obtained allow us to arrive at the following conclusions: if the time windows studied belong to a person who is sick, the complexity of the time series is low, the dimension of embedding is small (below 6), and the dimension of correlation is low (below 2). For healthy people, the dimension of embedding is higher (above 7), the correlation dimension is also higher (above 2). The transition from a completely healthy to a completely sick behavior and vice versa occurs continuously. If the temporary moving windows corresponding to stages of transit between completely healthy and completely diseased behavior had been included in the analysis, such marked differences in the distributions of the correlation dimensions in each of these cases do not happen suddenly. In general, the heart electrical signal of a healthy person is more complex than that of a sick person. Results similar to these have been obtained by other methods [25]. In the aforementioned work, the authors affirm that the results obtained by them show that the cardiac dynamics of a healthy subject is more complex and random compared to the same for a heart failure patient, whose dynamics is more deterministic [43]. Our results are more general because we have managed to classify temporary windows in "healthy" or "diseased" (even for the same person) in terms of the complexity of the time series from their embedding dimension or their correlation dimension.

Our work opens the possibility of observing with these tools patients, for example, under anesthesia and relaxation in the operating room and critical care unit where patients often have no possibility of spoken communication, opening another way to evaluate and monitor the increase or decrease in the complexity of the cardiac bioelectrical signal, with the future possibility to evaluate the severity of the sick state or the reduction of this, analyzing in real time the behavior of the dynamics of that bioelectrical signal by means of the observation of the loss or recovery of the dimension of that bioelectrical signal, which is getting a numeric value that suggests the severity of the patient.

The implications of this for the early warning of the episodes of dysfunction are clear. Once the embedding dimension or the correlation dimension falls below a preset threshold, we may consider that we are facing an emergency.

Finally, the algorithms must deliver the results in real time in order for early warning to be effective and this is a challenge that must be faced. The ever-increasing speed of digital devices will certainly help this goal. On the other hand, the thresholds for early warnings should be obtained from careful statistical experiments. 


\section{Author details}

Pedro Eduardo Alvarado Rubio ${ }^{1}$, Ricardo Mansilla Corona ${ }^{2}$, Lizette Segura Vimbela ${ }^{1 *}$, Alejandro González Mora ${ }^{1}$, Roberto Brugada Molina ${ }^{3}$, Cesar Augusto González López ${ }^{3}$ and Laura Yavarik Alvarado Avila ${ }^{4}$

*Address all correspondence to: merida_timucuy@yahoo.com.mx

1 Critical Care Unit Hospital Regional Lic., Adolfo López Mateos Social Security Institute for State Workers (ISSSTE), National Autonomous University of Mexico (UNAM), Mexico City, Mexico

2 Center for Interdisciplinary Research in the Sciences and Humanities (CEIICH), National Autonomous University of Mexico (UNAM), Mexico City, Mexico

3 Intensive Care Unit, Regional Hospital Lic, Adolfo López Mateos ISSSTE, Mexico City, Mexico

4 National Autonomous University of Mexico - Faculty of Veterinary Medicine, UNAM, Mexico City, Mexico

\section{References}

[1] Van der Pol B, Van der Mark J. Frequency demultiplication. Nature. 1927;120(3019):363364

[2] Van der Pol B, van der Mark J. The heart beat considered as a relaxation oscillator, and an electrical model of the heart. Philosophical Magazine (Supplements). 1928;6:763-775

[3] Babloyantz A, Destexhe A. Is the normal heart a periodic oscillator? Journal of Biological Cybernetics. 1988;58:203-211

[4] Goldberger A, West B. Applications of nonlinear dynamics to clinical cardiology. Annals of New York Academy of Sciences. 1987;504:195-213

[5] Glass L, Mackey M. From Clock to Chaos: The Rhythms of Life. Princeton, NJ: Princeton University Press; 1988

[6] Guevara MR, Glass L, Shrier A. Phase locking, period-doubling bifurcations, and irregular dynamics in periodically stimulated cardiac cells. Science. 1981;214:1350-1353

[7] Garfinkel A. A mathematics for physiology. American Journal Physiology. 1983;245:R455R466

[8] Ritzenberg A, Adam D, Cohen R. Period multiplying evidence for nonlinear behavior of the canine heart. Nature. 1984;307:159-161 
[9] Small M, et al. Automatic identification and recording of cardiac arrhythmia. Computers in Cardiology. 2000;27:355-358

[10] Wolf M, Varigos G, Hunt D, Sloman J. Sinus arrhythmia in acute myocardial infarction. Medical Journal of Australia. 1978;2:52-53

[11] Kitney RI, Rompelman O. The Study of Heart Rate Variability. Oxford: Clarendon Press; 1980

[12] Kleiger RE, Miller JP, Bigger JT, Moss AR. Multicenter Post-infarction Research Group. Decreased heart rate variability and its association with increased mortality after acute myocardial infarction. American Journal of Cardiology. 1987;59:256-262

[13] Lombardi F, Porta A, Marzegalli M, Favale S, Santini M, Vincenti A, De Rosa A, and participating investigators of ICD-HRV Italian Study Group. Heart rate variability patterns before ventricular tachycardia onset in patients with an implantable cardioverter defibrillator. American Journal of Cardiology. 2000;86:959-963

[14] FitzHugh R. Impulses and physiological states in theoretical models of nerve membrane. Biophysical Journal. 1961;1:445-466

[15] Nagumo J, et al. An active pulse transmission line simulating nerve axon. Proceedings of IEEE. 1962;50:2061-2070

[16] Small M, et al. Automatic identification and recording of cardiac arrhythmia. Computers in Cardiology. 2000;27:355-358

[17] Venkatesh M, et al. Association of short term heart rate spectral power with onset of spontaneous ventricular tachycardia or ventricular fibrillation. Computers in Cardiology. 1998;25:101-104

[18] Ervin S, Lewis A. Necessity of noise in physiology and medicine. Computer Methods and Programs in Biomedicine. 2013;111:459-470

[19] Small M, et al. Deterministic nonlinearity in ventricular fibrillation. Chaos. 2000;10:268-277

[20] Zhang XSh, et al. Detecting ventricular tachycardia and fibrillation by complexity measure. IEEE Transactions on Biomedical Engineering. 1999;46:548-555

[21] Hon EH, Lee ST. Electronic evaluation of the fetal heart rate. VIII. Patterns preceding fetal death, further observations. American Journal of Obstetrics and Gynecology. 1963 Nov $15 ; 87: 814-826$

[22] Kobayashi M, Musha T. 1/f fluctuation of heartbeat period. IEEE Transactions on Biomedical Engineering. 1982;29:456-457

[23] Goldberger A, Rigney D, Mietus J, Antman E, Greenwald S. Nonlinear dynamics in sudden cardiac death syndrome: Heart rate oscillations and bifurcations. Experientia. 1988;44:983-987

[24] Goldberger A. Is the normal heartbeat chaotic or homeostatic? News in Physiological Science. 1991;6:87-91 
[25] Voss A, Schulz S, Schroeder R, Baumert M, Caminal P. Methods derived from nonlinear dynamics for analyzing heart rate variability. Philosophical Transactions of the Royal Society A. 2009;367:277-296

[26] Norris PR, Anderson SM, Jenkins JM, Williams AE, Morris Jr, JA. Heart rate multiscale entropy at three hours predicts hospital mortality in 3,154 trauma patients. Shock. 2008;30:17-22

[27] Bär K-J, Boettger MK, Koschke M, Schulz S, Chokka P, Yeragani VK, Voss A. Non-linear complexity measures of heart rate variability in acute schizophrenia. Clinical Neurophysiology. 2007;118:2009-2015

[28] Bar KJ, Letzsch A, Jochum T, Wagner G, Greiner W, Sauer H, et al. Loss of efferent vagal activity in acute schizophrenia. Journal of Psychiatric Research. 2005;39:519-527

[29] Hejjel L, Gál I. Heart rate variability analysis. Acta Physiologica Hungarica. 2001;88:219230

[30] Amaral LAN, Goldberger AL, Ivanovc PCh, Stanley HE. Modeling heart rate variability by stochastic feedback. Computer Physics Communications. 1999;121-122:126-128

[31] Berne RM, Levy MN. Cardiovascular Physiology. 6th ed. St. Louis: C.V. Mosby; 1996

[32] Takens F. Detecting strange attractors in turbulence. In: Rand D, Young L, editors. Springer Lecture Notes in Mathematics. Vol. 898. 1981. pp. 366-381

[33] Small M. Applied Nonlinear Time Series Analysis. Applications in Physics, Physiology and Finance. World Scientific Publishing; 2005

[34] Shannon C. A mathematical theory of communication. The Bell System Technical Journal. 1948;27:3

[35] Fraser A, Swinney H. Independent coordinates for strange attractors from mutual information. Physical Review A. 1986;33(2):1134-1140

[36] Celluci C, et al. Statistical validation of mutual information calculations: Comparison of alternative numerical algorithms. Physical Review E. 2005;71:066208

[37] Kaplan DT, Furman MI, Pincus SM, Ryan SM, Lipsitz LA, Goldberger AL. Aging and the complexity of cardiovascular dynamics. Biophysical Journal. 1991;59(4):945-949

[38] Malliani A, Pagani M, Lombardi F, Cerutti S. Cardiovascular neural regulation explored in the frequency domain. Circulation. 1991;84(2):482-492

[39] Pagani M, Lombardi F, Guzzetti S, et al. Power spectral analysis of heart rate and arterial pressure variabilities as a marker of sympatho-vagal interaction in man and conscious dog. Circulation Research. 1986;59(2):178-193

[40] Malliani A, Montano N. Heart rate variability as a clinical tool. Italian Heart Journal. 2002;3(8):439-445 
[41] Goldberger JJ. Sympathovagal balance: How should we measure it? American Journal of Physiology - Heart and Circulatory Physiology. 1 April 1999;276(4)

[42] Schmidt H, et al. Impaired chemoreflex sensitivity in adult patients with multiple organ dysfunction syndrome: The potential role of disease severity. Intensive Care Medicine. 2004;30:665-672

[43] Mukherlee S, et al. Can complexity decrease in congestive heart failure? Physica A. 2015;439:93-102 
